\title{
Why Teenage Risk Taking Behavior is Going Too High? \\ Sushma Suri
}

Department of Psychology, Jamia Millia Islamia University, New Delhi, India

*Corresponding author: Sushma Suri, Department of Psychology, Jamia Millia Islamia University, New Delhi, India, Tel: 9999755693; E-mail: ssuri@jmi.ac.in

Received date: Dec 15, 2015; Accepted date: Dec 28, 2015; Published date: Dec 31, 2015

Copyright: (c) 2015 Suri S. This is an open-access article distributed under the terms of the Creative Commons Attribution License, which permits unrestricted use, distribution, and reproduction in any medium, provided the original author and source are credited.

\begin{abstract}
This article proposes Decision making (Fuzz trace theory) and Evolutionary theories and their role within a new framework of investigations that have been applied to the Teen health risk behavior. The trace theory argues that how people represent, retrieve, and process information when they make decisions, and how decision making changes with development. Gist representations often incorporate emotion including valence, arousal, feeling states, and discrete emotions and Emotion determines whether gist or verbatim representations are processed. Trace theory also indicates that Intuition is developmentally advanced and that emotion is integral to intuition just opposite to classical decision making theory that assumes development progresses from hot intuitive thinking to cold calculation bypassing emotion. On the other hand evolutionary model emphasizes that natural selection shaped human neurobiological mechanisms. Most important, these responses are not arbitrary but function adaptively to calibrate developmental and behavioral strategies to match that environment. These theories promise to be valuable as comprehensive descriptive reference material for health professionals, psychologists, academicians and particularly for parents. And have broad implications for designing interventions for high-risk youth and suggests new directions for research.
\end{abstract}

Keywords: Decision making; Evolutionary theory; Health risk behavior; Adolescents

\section{Introduction}

The study of adolescent risk taking behavior gained popularity in 1990 's as it become increasingly evident that majority of morbidity and mortality life was behavioral in origin. The term risk taking behavior has been defined behaviors that increase the likelihood of adverse physical, social or psychological consequences [1]. These behaviors are usually established during childhood, and are preventable. Overviewing the risk taking behavior one can observe that it include a number of potentially health damaging behavior like alcohol/ drug use, unintentional injuries/violence behavior (including suicide), tobacco use, unhealthy dietary intake, irrational driving, physical inactivity and sexual behavior which contribute to the leading causes of death and disability among adults and youth [2]. The World Health Organization estimates that $70 \%$ of premature deaths among adults are due to behavioral patterns that emerge in adolescence, including smoking, violence, and sexual behavior. The author begins with describing the explanatory frame work for adolescent risk behaviour derived from different theoretical perspective. The Decision making theory stated that how people represent, retrieve, and process information when they make decisions, and how decision making changes with development $[3,4]$. According to this theory advanced judgment and decision making is based on simple, gist mental representations of choices ("fuzzy" memory traces) as opposed to more detailed, quantitative representations (verbatim memory traces). Gist refers to the meaning an individual extracts from information (i.e., the semantic representation), which reflects the individual's knowledge, understanding, culture, and developmental level [5,6]. As individuals develop and acquire greater expertise in a domain, their decisions tend to be based on the meaning of the information in contrast to its verbatim details [7]. Laboratory experiments with children, adolescents, and adults have confirmed the prediction that the decision making becomes less computational and more intuitive as development proceeds [4,8-13]. Studies have shown that younger adolescents tend to base decisions on verbatim representations of details, trading off the amount of risk against the amount of rewards, and thus take more risks, compared to older adolescents and adults [6]. While, mature decision makers tend to rely on the bottom-line gist that potentially catastrophic risks should be avoided under ordinary circumstances [14].

Children with their limited experience often face the nuanced situations. The common adolescent rationale "all my friends are doing it" is understandable because negative outcomes are usually rare, especially if one's experience is limited. Study by [15] on youth perceptions of smoking supports this analysis. The meaning of smoking to young smokers is positive; smoking is interpreted as fun, exciting, and something to do with friends. The negative consequences of smoking are not yet attached to their gist of the behavior. In addition, as their positive evaluations of the behavior increase, negative evaluations diminish [16]. Decision making under the influence of positive feeling states occurs more quickly and efficiently, often relying on stereotypes and other heuristics, than decision making under neutral or negative feeling states $[17,18]$ which do not involve necessarily a lowered motivation to process information [19]. Discrete emotional states, such as anger, sadness, joy, and fear which encompass relatively distinct combinations of feeling states, arousal patterns, and cognitive appraisal tendencies (among other features), have a distinct impact on judgment and decision making [20-22].

As far the evolutionary model is concerned that natural selection shaped human neurobiological mechanisms to detect and respond to the fitness-relevant costs and benefits afforded by different environments. Most important, these responses are not arbitrary but function adaptively to calibrate developmental and behavioral strategies to match those environments [23]. In particular, an 
Page 2 of 3

evolutionary perspective contends that both stressful and supportive environments have been part of the human experience throughout our history, and that developmental systems shaped by natural selection respond adaptively to both kinds of contexts [24]. According to life history (LH) theory, children's brains and bodies tend to respond to dangerous or unpredictable environments by growing up fast and living for the here and now [25-27]. Viewed from within this framework, the adolescent who responds to a dangerous environment by developing insecure attachments, adopting an opportunistic interpersonal orientation, engaging in a range of externalizing behaviors, and sustaining an early sexual debut is no less functional than the adolescent who responds to a well-resourced and supportive social environment by developing the opposing characteristics and orientations $[24,28]$. Children and young adolescents who tend to be rejected or ignored by peers are those most likely to form coalitions with other high-risk children and engage in "deviancy training" (i.e., giving attention and rewards for talk about engaging in deviant behavior) in the context of the playground [29] as well as in their adolescent friendships. There is a clear process through which middle school children who are harassed by their peers into marginal positions in status hierarchies come to associate with deviant peers and engage in progressively higher levels of risky and antisocial behavior overtime [30], in a study of 206 male youth [31] behavioral observations of deviancy training were collected at ages 13-14, 15-16, and 17-18. Most critically, observations at age 13-14 were the most prognostic of delinquent and antisocial behavior in young adulthood. These data further highlight puberty and early adolescence as an inflection point in trajectories of high-risk behavior in young men. Brain Development During Adolescence On the basis of structural brain-imaging studies conducted during the past decade, we now know that significant increases in white matter (which represent fiber growth and myelination) take place during adolescence and continues into the early 20 s [32].

\section{Conclusion}

The issue of risky adolescent behavior is really a complex issue and any intervention aimed at reducing it (or ameliorating its consequences) faces obstacles and complications. Risk taking in adolescent provides an excellent testing ground for evaluating the potential of decision making and evolutionary approaches to human development. Fzzy-trace theory is the only developmental theory that identifies advanced reasoning with the gist-based processes of intuition. The theory argued, by inculcating stable gist representations that encode danger (e.g., the risk associated with an unsupervised party or with a man approaching a young girl in a mall) and by practicing retrieval of simple values and principles until they are evoked automatically in relevant situations. Developing such educated intuitions should shield adolescent risky decision making somewhat from the strong emotions that infuse this time of life. These intervention approaches are aimed at changing how adolescents make decisions about risk. Intervention approaches that aim to effect change at different levels such as within a community, school, family, neighborhood etc. The gist of new stimuli can be manipulated by varying the salience of positive or negative information. By teaching adolescents how to quickly and automatically encode the negative valence of myriad situations that threaten sexual health, adolescents are more likely to avoid these situations $[6,14]$ more likely to influence judgments in relatively ambiguous and complex situations which require constructive cognitive processing [33]. Distracted by multiple representations, they are less likely to focus on theinformation that promotes health, and discourages unhealthy risk taking. Recognizing that younger adolescents are especially lacking in inhibitory control, intervention approaches may take a preemptive approach to deal with this group.

On the other hand the evolutionary model supplements, extends, and amends the standard develop mental psychopathology approach in two interconnected ways. First, the evolutionary model delivers a detailed theoretical foundation for understanding the meaning and manifestations of risky behavior. Second, it can inform prevention and treatment programs by highlighting key variables, indicating ways to maximize program effectiveness, revealing potential pitfalls and tradeoffs, increasing the realism of the intervention goals, and in some cases suggesting truly novel approaches and solutions.

However by implementing different intervention programs and strategies we can provide a positive platform to deal with the situation. Interventions need to work within the goal structures and motivation of adolescents to substitute effective, evolutionarily and informed prosocial strategies that yield outcomes and incentives that are comparable to those achieved through risk taking, delinquency, or antisocial behavior.

An evolutionary analysis explains the functional significance of puberty-specific changes. A guiding assumption of the evolutionary model is that understanding the functions of adolescence is essential to explaining why adolescents engage in risky behavior. Indeed, adolescence may be a phase of the life span that historically had great influence on fitness and was thus under intense selection pre demands that make their behavior risky without any consideration.

Recognizing that younger adolescents are especially lacking in inhibitory control, intervention approaches may take a preemptive strategy to deal with situations.The concept of environmental mismatch has also been invoked to explain adolescent risky behavior. Hence, a need to establish some evolutionarily guided treatment programs which particularly focus on restoring ancient patterns of diet, sleep, exercise, natural light exposure, and social connectedness [34]. Interventions that attempt to restore more natural relationships between adolescents, adults and younger children may have powerful effects on social development. Prevention and treatment programs need to address the causative environmental conditions which provoke adolescent to behave in a negative manner. And by altering the social context induce an understanding that they can lead longer, healthier, more predictable lives.

Culture, media and social climate also need to be addressed through broader social policy change. Keeping in mind the importance of positive experiences during transition periods of the child should be appropriately addressed within intervention program to reduce marginalization, social exclusion and the vulnerability of young people during transition periods.

\section{Limitations}

The present study is based on review of literature hence restricted to what was reported and discussed in review of literature. As a result we are limited in terms of identifying very important determinants of risk behavior of adolescents. 
Page 3 of 3

\section{References}

1. Resnick MD, Bearman PS, Blum RW, Bauman KE, Harris KM, et al (1997) Protecting adolescents from harm. Findings from the National Longitudinal Study on Adolescent Health. JAMA 278: 823-832.

2. Centers for Disease Control and Prevention (CDC) $(2004,2007 \mathrm{c})$ Leading cause of death in males United States.

3. Reyna VF (2004) How people make decisions that involve risk: A dualprocesses approach. Current Directions in Psychological Science 13 60-66.

4. Reyna VF, Lloyd FJ, Brainerd CJ (2003) Memory, development, and rationality: An integrative theory of judgment and decision making. In Schneider SL, Shanteau J (eds.) Emerging perspectives on judgment and decision research. Cambridge University Press, New York.

5. Reyna VF, Brainerd CJ (1998) Fuzzy-trace theory and false memory: New frontiers. J Exp Child Psychol 71: 194-209.

6. Reyna VF, Farley F (2006) Risk and Rationality in Adolescent Decision Making: Implications for Theory, Practice, and Public Policy. Psychol Sci Public Interest 7: 1-44.

7. Reyna VF, Kiernan B (1994) The development of gist versus verbatim memory in sentence recognition: Effects of lexical familiarity, semantic content, encoding instructions, and retention interval. Developmenta Psychology 30: 178-191.

8. Reyna VF (1996) Conceptions of memory development, with implications for reasoning and decision making. Annals of Child Development 12: 87-118.

9. Reyna VF, Brainerd CJ (1991b) Fuzzy-trace theory and framing effects in choice: Gist extraction, truncation, and conversion. Journal of Behavioral Decision Making 4: 249-262.

10. Reyna VF, Brainerd CJ (1993) Fuzzy memory and mathematics in the classroom. In: Davies G M, Logie R H, (eds.) Memory in everyday life. Elsevier Science Publishers, Amsterdam, Netherlands.

11. Reyna VF, Brainerd CJ (1994) The origins of probability judgment: A review of data and theories. In: Wright G, Ayton P, editors. Subjective probability. John Wiley \& Sons, Oxford, England.

12. Reyna VF, Brainerd CJ (1995a) Fuzzy-trace theory: An interim synthesis Learning and Individual Differences 7: 1-75.

13. Reyna VF, Ellis SC (1994) Fuzzy-trace theory and framing effects in children's risky decision making. Psychological Science 5: 275-279.

14. Reyna VF, Adam MB, Poirier K M, LeCroy CW, Brainerd CJ (2005) Risky decision making in childhood and adolescence: A fuzzy-trace theory approach. In: Jacobs JE, Klaczynski PA (eds.) The development of judgment and decision making in children and adolescents. Lawrence Erlbaum Associates Publishers Mahwah, NJ.

15. Slovic P (2001) Cigarette smokers: Rational actors or rational fools? In: Slovic P (eds.) Smoking: Risk, perception and policy. Sage Publications Inc, Thousand Oaks, CA.

16. Slovic P, Finucane ML, Peters E, MacGregor DG (2004) Risk as analysis and risk as feelings: Some thoughts about affect, reason, risk, and rationality. Risk Anal 24: 311-322.
17. Brainerd CJ, Gordon LL (1994) Development of verbtaim and gist memory for number. Developmental Psychology 30: 163-177.

18. Isen AM (2001) An influence of positive affect on decision making in complex situations: Theoretical issues with practical implications. Journal of Consumer Psychology 11: 75-85.

19. Storbeck J, Clore GL (2005) With sadness comes accuracy; with happiness, false memory: Mood and the false memory effect. Psychol Sci 16: 785-791.

20. Abe JA, Izard CE (1999) The developmental functions of emotions: An analysis in terms of differential emotions theory. Cognition and Emotion 13: 523-549.

21. Frijda NH (1986) The emotions. University Press, Cambridge, England.

22. Lazarus RS (1991) Emotion and adaptation. Oxford University Press, New York.

23. Ellis BJ (2004) Timing of pubertal maturation in girls: an integrated life history approach. Psychol Bull 130: 920-958.

24. Ellis BJ, Boyce WT, Belsky J, Bakermans-Kranenburg MJ, van IJzendoorn MH (2011) Differential susceptibility to the environment: An evolutionary-neurodevelopmental theory. Development and Psychopathology 23: 7-28.

25. Ellis BJ, Figueredo AJ, Brumbach BH, Schlomer GL (2009) Fundamental dimensions of environmental risk: The impact of harsh versus unpredictable environments on the evolution and development of life history strategies. Human Nature 20: 204-268.

26. Nettle D (2010) Dying young and living fast: Variation in life history across English neighborhoods. Behavioral Ecology 21: 387-395.

27. Quinlan RJ (2007) Human parental effort and environmental risk. Proc Biol Sci 274: 121-125.

28. Belsky J, Steinberg L, Draper P (1991) Childhood experience, interpersonal development and reproductive strategy: An evolutionary theory of socialization. Child Development 62: 647-670.

29. Snyder J, Schrepferman L, Oeser J, Patterson G, Stoolmiller M, et al. (2005) Deviancy training and association with deviant peers in young children: Occurrence and contribution to early-onset conduct problems. Development and Psychopathology 17: 397-413.

30. Rusby JC, Forrester KK, Biglan A, Metzler CW (2005) Relationships between peer harassment and adolescent problem behaviors. Journal of Early Adolescence 25: 453-477.

31. Dishion TJ, Nelson SE, Bullock BM (2004) Premature adolescent autonomy: parent disengagement and deviant peer process in the amplification of problem behaviour. J Adolesc 27: 515-530.

32. Hazen E, Schlozman S, Beresin E (2008) Adolescent psychological development: a review. below Pediatr Rev 29: 161-167.

33. Forgas JP, Vargas PT (2000) The effects of mood on social judgment and reasoning. In: Lewis M, Haviland JM (eds.) Guilford Press, New York.

34. Ilardin SS (2009) The depression cure: The 6-step program to beat depression without drugs. MA: Da Capo, Cambridge. 